\title{
Existence of Solutions to the Boundary Value Problems for a Class of P-Laplacian Equations at Resonance
}

\author{
Lina Zhou ${ }^{1} \&$ Weihua Jiang ${ }^{2}$ \\ ${ }^{1}$ College of Mathematics and Information Science, Hebei Normal University, Shijiazhuang 050024 Hebei, China \\ ${ }^{2}$ College of Science,Hebei University of Science and Technology, Shijiazhuang 050018, Hebei, China \\ Correspondence: Lina Zhou. E-mail: lnazhou@163.com
}

Received: March 8, 2016 Accepted: April 4, 2016 Online Published: May 18, 2016

doi:10.5539/jmr.v8n3p37 URL: http://dx.doi.org/10.5539/jmr.v8n3p37

\begin{abstract}
By the generalizing the extension of the continuous theorem of Ge and Ren and constructing suitable Banach spaces and operators, we investigate the existence- solutions to the boundary value problems for a class of p-Laplacian equations. Finally an example is given to illustrate our results.
\end{abstract}

Keywords: continuous theorem, resonance, p-Laplacian equations, boundary value problem

\section{Introduction}

In this paper,we will study the boundary value problem

$$
\left\{\begin{array}{l}
\left(\varphi_{p}\left(u^{\prime \prime}\right)\right)^{\prime}(t)=f\left(t, v, v^{\prime}, v^{\prime \prime}\right) \\
\left(\varphi_{p}\left(v^{\prime \prime}\right)\right)^{\prime}(t)=g\left(t, u, u^{\prime}, u^{\prime \prime}\right) \\
u(0)=u^{\prime \prime}(0)=0, v(0)=v^{\prime \prime}(0)=0 \\
u^{\prime}(1)=\int_{0}^{1} k_{1}(t) u^{\prime}(t) d t, v^{\prime}(1)=\int_{0}^{1} k_{2}(t) v^{\prime}(t) d t
\end{array}\right.
$$

where $\varphi_{p}(s)=|s|^{p-2} s, p>1, \int_{0}^{1} k_{i}(t) d t=1, i=1,2$

In this paper,we will always suppose that

$\left(H_{1}\right) k_{i}(t) \in L^{1}[0,1]$ are nonnegative and $\left\|k_{i}\right\|_{1}=1$, where $\left\|k_{i}\right\|_{1}=\int_{0}^{1}\left|k_{i}(t)\right| d t, i=1,2$.

$\left(H_{2}\right) f(t, u, v, w), g(t, u, v, w)$ is continuous in $[0,1] \times R^{3}$

\section{Preliminaries}

Definition 2.1 Let $X$ and $Y$ be a two Banach spaces with norms $\|\cdot\|_{X},\|\cdot\|_{Y}$,respectively.A continuous operator $M$ : $X \cap$ dom $M \longrightarrow$ Yis said to be quasi-linear if

(i) $\operatorname{Im} M:=M(X \cap \operatorname{dom} M)$ is a closed subset of $Y$,

(ii)KerM $:=\{x \in X \cap \operatorname{dom} M: M x=0\}$ is linearly homeomorphic to $R^{n}, n<\infty$.

where dom $M$ denote the domain of operator $M$.

Let $X_{1}=\operatorname{Ker} M$ and $X_{2}$ be complement space of $X_{1}$ in $X$, then $X=X_{1} \bigoplus X_{2}$. Let $P: X \longrightarrow X_{1}$ be a projector and $\Omega \subset X$ an open and bounded set with the origin $\theta \in \Omega$

Definition2.2 Suppose $N_{\lambda}: \bar{\Omega} \rightarrow Y, \lambda \in[0,1]$ is a continuous and bounded operator.Denote $N_{1}$ by $N_{\text {.Let }} \Sigma_{\lambda}=$ $\left\{x \in \bar{\Omega} \cap\right.$ dom $\left.M: M x=N_{\lambda} x\right\} . N_{\lambda}$ is said $M$-quasi-compact in $\bar{\Omega}$ if there exists a vector subspace $Y_{1}$ of $Y$ satisfying $\operatorname{dim} Y_{1}=\operatorname{dim} X_{1}$ and two operators $Q, R$ with $Q: Y \rightarrow Y_{1}, Q Y=Y_{1}$, being continuous, bounded,and satisfying $Q(I-Q)=$ $0, R: \bar{\Omega} \times[0,1] \rightarrow X_{2} \cap$ dom $M$ continuous and compact such that for $\lambda \in[0,1]$,

(a) $(I-Q) N_{\lambda}(\bar{\Omega}) \subset \operatorname{Im} M \subset(I-Q) Y$,

(b) $Q N_{\lambda} x=\theta, \lambda \in(0,1) \Leftrightarrow Q N x=\theta$,

(c) $R(\cdot, 0)$ is zero operator and $\left.R(\cdot, 0)\right|_{\Sigma}=\left.(I-P)\right|_{\Sigma}$,

(d) $M[P+R(\cdot, 0)]=(I-Q) N_{\lambda}$

Theorem 2.1 Let $X$ and $Y$ be two Banach spaces with norms $\|\cdot\|_{X},\|\cdot\|_{Y}$, respectively, and $\Omega \subset X$ be an open and bounded nonempty set.Suppose

$$
M: X \cap \operatorname{dom} M \rightarrow Y
$$


is a quasi-linear operator and that $N_{\lambda}: \bar{\Omega} \rightarrow Y, \lambda \in[0,1]$ is M-quasi-compact.In addition,if the following conditions hold:

$\left(C_{1}\right) M x \neq N_{\lambda} x, \forall x \in \partial \Omega \cap \operatorname{dom} M, \lambda \in(0,1)$,

$\left(C_{2}\right) \operatorname{deg}\{J Q N, \Omega \cap \operatorname{KerM}, 0\} \neq 0$

then the abstract equation $M x=N x$ has at least one solution in dom $\cap \cap \bar{\Omega}$, where $N=N_{1}, J: \operatorname{Im} Q \rightarrow \operatorname{KerM}$ is a homeomorphism with $J(\theta)=\theta$.

Proof. The proof is similar to the one of lemma 2.1 and Theorem 2.1 in [Ge et al., 2004].

We can easily get the following inequalities.

Lemma2.1 For any $u, v \geq 0$, we have

(1) $\varphi_{p}(u, v) \leq \varphi_{p}(u)+\varphi_{p}(v), 1<p \leq 2$.

(2) $\varphi_{p}(u, v) \leq 2^{p-2}\left(\varphi_{p}(u)+\varphi_{p}(v)\right), p \geq 2$.

In the following,we will always suppose that $q$ satisfies $\frac{1}{p}+\frac{1}{q}=1$.

\section{Main Results}

Let $X=C^{2}[0,1] \times C^{2}[0,1]$ with norm $\|(u, v)\|=\|u\|+\|v\|$, where $\|u\|=\max \left\{\|u\|_{\infty},\left\|u^{\prime}\right\|_{\infty},\left\|u^{\prime \prime}\right\|_{\infty}\right\}$,

$Y=C[0,1] \times C[0,1] \times C[0,1] \times C[0,1]$ with norm $\left\|\left(y_{1}, y_{2}, y_{3}, y_{4}\right)\right\|=\max \left\{\left\|y_{1}\right\|_{\infty},\left\|y_{2}\right\|_{\infty},\left\|y_{3}\right\|_{\infty},\left\|y_{4}\right\|_{\infty}\right\}$ with

$\|y\|_{\infty}=\max _{t \in[0,1]}|y(t)|$. We know that $(X,\|\cdot\|)$ and $(Y,\|\cdot\|)$ are Banach spaces.

Define operators $M: X \cap \operatorname{dom} M \longrightarrow Y, N_{\lambda}: X \rightarrow Y$ as follows:

$$
M(u, v)=\left(\begin{array}{c}
\left(\varphi_{p}\left(u^{\prime \prime}\right)\right)^{\prime}(t) \\
\left(\varphi_{p}\left(v^{\prime \prime}\right)\right)^{\prime}(t) \\
T_{1}\left(\varphi_{p}\left(u^{\prime \prime}\right)\right)^{\prime}(t) \\
T_{2}\left(\varphi_{p}\left(v^{\prime \prime}\right)\right)^{\prime}(t)
\end{array}\right), N_{\lambda}(u, v)=\left(\begin{array}{c}
\lambda f\left(t, v(t) v^{\prime}(t), v^{\prime \prime}(t)\right) \\
\lambda g\left(t, u(t), u^{\prime}(t), u^{\prime \prime}(t)\right) \\
0 \\
0)
\end{array}\right)
$$

where $T_{i} y=c_{i}, i=1,2 . y \in C[0,1], c_{1}, c_{2}$ satisfy

$$
\begin{gathered}
\int_{0}^{1} k_{i}(t) \int_{t}^{1} \varphi_{q}\left(\int_{0}^{s}\left(y(r)-c_{i}\right) d r\right) d s d t=0 \\
\operatorname{dom} M=\left\{(u, v) \in X \mid \varphi_{p}\left(u^{\prime \prime}\right), \varphi_{p}\left(v^{\prime \prime}\right) \in C^{1}[0,1], u(0)=u^{\prime \prime}(0)=v(0)=v^{\prime \prime}(0)=0\right\}
\end{gathered}
$$

Lemma3.1 For $y \in C[0,1]$, there is only one constant $c_{i} \in R$ such that $T_{i} y=c_{i}$, with $\left|c_{i}\right| \leq\|y\|_{\infty}$, and $T_{i}: C[0,1] \rightarrow R$, $i=1,2$. are continuous .

The proof is similar to Lemma 3.1 in [Weihua, 2014].

It is clear that $(u, v) \in \operatorname{dom} M$ is a solution if and only if it satisfies $M(u, v)=N(u, v)$ where $N=N_{1}$.For convenience,

$\operatorname{let}(a, b, c, d)^{L}=\left(\begin{array}{l}a \\ b \\ c \\ d\end{array}\right)$

Lemma3.2 $M$ is a quasi-linear operator.

Proof. It is easy to get $\operatorname{Ker} M=\left\{\left(b_{1} t, b_{2} t\right) \mid b_{1}, b_{2} \in R\right\}:=X_{1}$.

For $(u, v) \in X \cap \operatorname{dom} M$, if $M(u, v)=\left(y_{1}, y_{2}, c_{1}, c_{2}\right)^{L}$,then $c_{1}, c_{2}$ satisfy (3.1). On the other hand if $y_{i} \in C[0,1], T_{i} y_{i}=c_{i}$, $i=1,2$, take

$$
\left(\begin{array}{c}
u(t) \\
v(t)
\end{array}\right)=\left(\begin{array}{c}
\int_{0}^{t}(t-s) \varphi_{q}\left(\int_{0}^{s} y_{1}(r) d r\right) d s \\
\int_{0}^{t}(t-s) \varphi_{q}\left(\int_{0}^{s} y_{2}(r) d r\right) d s
\end{array}\right) .
$$

We can get $(u, v) \in X \cap \operatorname{dom} M$ and $M(u, v)=\left(y_{1}, y_{2}, c_{1}, c_{2}\right)^{L}$, then $c_{1}, c_{2}$. Thus

$$
\operatorname{Im} M=\left\{\left(y_{1}, y_{2}, c_{1}, c_{2}\right)^{L} \mid y \in C[0,1], c_{1}, c_{2} \text { satisfy (3.1) } .\right.
$$

By the continuity of $T_{i}, i=1,2$, we see that $\operatorname{Im} M \subset Y$ is closed.So, $M$ is quasi-linear.The proof is completed.

Lemma3.3 $T_{i}(c)=c, T_{i}(y+c)=T_{i}(y)+c, T_{i}(c y)=c T_{i}(y), i=1,2, c \in R, y \in C[0,1]$ 
Proof. The proof is simple.Therfore we omit it.

Take a projector $P: X \rightarrow X_{1}$ and an operator $Q: Y \rightarrow Y_{1}$ as follows:

$$
(P(u, v))(t)=\left(u^{\prime}(0) t, v^{\prime}(0) t\right), Q\left(y_{u}, y_{v}, y_{1}, y_{2}\right)^{L}=\left(0,0, T_{1} y_{1}-T_{1} y_{u}, T_{2} y_{2}-T_{2} y_{v}\right)^{L}
$$

where $Y_{1}=\left\{\left(0,0, c_{1}, c_{2}\right)^{L} \mid c_{i} \in R, i=1.2\right\}$. Obviously $Q Y=Y_{1}$ and $\operatorname{dim} Y_{1}=\operatorname{dim} X_{1}$.

By the continuity and boundedness of $T_{i}, i=1,2$,we can easily see that $Q$ is continuous and bounded in $Y$.It follows Lemma3.3 that $Q(I-Q)\left(y_{u}, y_{v}, y_{1}, y_{2}\right)^{L}=(0,0,0,0)^{L}, y_{u}, y_{v}, y_{1}, y_{2} \in C[0,1]$

Define a operator $R: X \times[0,1] \rightarrow X_{2}$

$$
R(u, v, \lambda)(t)=\left(\begin{array}{c}
\int_{0}^{t}(t-s) \varphi_{q}\left(\int_{0}^{s} \lambda f\left(r, v(r), v^{\prime}(r), v^{\prime \prime}(r)\right) d r\right) d s \\
\int_{0}^{t}(t-s) \varphi_{q}\left(\int_{0}^{s} \lambda g\left(r, u(r), u^{\prime}(r), u^{\prime \prime}(r)\right) d r\right) d s
\end{array}\right),
$$

where $\operatorname{Ker} M \bigoplus X_{2}=X$.By $\left(H_{2}\right)$ and the Arzela-Asscoli theorem,we can get $R: \bar{\Omega} \times[0,1] \rightarrow X_{2}$ is continuous and compact,where $\Omega \subset X$ is a bounded set.

Lemma3.4 Assume that $\Omega \in X$ is an open bounded set.Then $N_{\lambda}$ is $M$-quasi-compact in $\bar{\Omega}$.

Proof. It is clear that $\operatorname{Im} P=\operatorname{Ker} M, Q N_{\lambda}(u, v)=\theta \Leftrightarrow Q N(u, v)=\theta$ and $R(\cdot, \cdot, 0)=0$. for $(u, v) \in \bar{\Omega}$,

$$
\begin{aligned}
(I-Q) N_{\lambda}(u, v)= & \left(\begin{array}{c}
\lambda f\left(t, v(t), v^{\prime}(t), v^{\prime \prime}(t)\right) \\
\lambda g\left(t, u(t), u^{\prime}(t), u^{\prime \prime}(t)\right) \\
0 \\
0
\end{array}\right)-\left(\begin{array}{c}
0 \\
0 \\
T_{1}\left(\lambda f\left(t, v(t), v^{\prime}(t), v^{\prime \prime}(t)\right)\right) \\
T_{2}\left(\lambda g\left(t, u(t), u^{\prime}(t), u^{\prime \prime}(t)\right)\right.
\end{array}\right) \\
& =\left(\begin{array}{c}
\lambda f\left(t, v(t), v^{\prime}(t), v^{\prime \prime}(t)\right) \\
\lambda g\left(t, u(t), u^{\prime}(t), u^{\prime \prime}(t)\right) \\
T_{1}\left(\lambda f\left(t, v(t), v^{\prime}(t), v^{\prime \prime}(t)\right)\right) \\
T_{2}\left(\lambda g\left(t, u(t), u^{\prime}(t), u^{\prime \prime}(t)\right)\right.
\end{array}\right) \in \operatorname{Im} M .
\end{aligned}
$$

Since $\operatorname{Im} M \subset \operatorname{Ker} Q$, and $y=Q y+(I-Q) y$, we obtain $\operatorname{Im} M \subset(I-Q) Y$.Thus $(I-Q) N_{\lambda}(\bar{\Omega}) \subset \operatorname{Im} M \subset(I-Q) Y$. For $(u, v) \in \Sigma_{\lambda}=\left\{(u, v) \in \bar{\Omega} \cap \operatorname{dom} M \mid M(u, v)=N_{\lambda}(u, v)\right\}$

$$
\begin{aligned}
R(u, v, \lambda)= & \left(\begin{array}{c}
\int_{0}^{t}(t-s) \varphi_{q}\left(\int_{0}^{s} \lambda f\left(r, v(r), v^{\prime}(r), v^{\prime \prime}(r)\right) d r\right) d s \\
\int_{0}^{t}(t-s) \varphi_{q}\left(\int_{0}^{s} \lambda g\left(t, u(t), u^{\prime}(t), u^{\prime \prime}(t)\right) d r\right) d s
\end{array}\right) \\
= & \left(\begin{array}{c}
\left.\int_{0}^{t}(t-s) \varphi_{q}\left(\int_{0}^{s} \varphi_{p}\left(u^{\prime \prime}\right)\right)^{\prime}(r) d r\right) d s \\
\left.\int_{0}^{t}(t-s) \varphi_{q}\left(\int_{0}^{s} \varphi_{p}\left(v^{\prime \prime}\right)\right)^{\prime}(r) d r\right) d s
\end{array}\right) \\
& =\left(\begin{array}{c}
u(t)-u^{\prime}(0) t \\
v(t)-v^{\prime}(0) t
\end{array}\right)=(I-P)(u, v) .
\end{aligned}
$$

i.e. Definition 2.2(c)holds.For $u \in \bar{\Omega}$, we have

$$
M[P(u, v)+R(u, v, \lambda)]=\left[\begin{array}{c}
\lambda f\left(r, v(r), v^{\prime}(r), v^{\prime \prime}(r)\right) \\
\lambda g\left(t, u(t), u^{\prime}(t), u^{\prime \prime}(t)\right) \\
T_{1}\left(\lambda f\left(t, v(t), v^{\prime}(t), v^{\prime \prime}(t)\right)\right) \\
T_{2}\left(\lambda g\left(t, u(t), u^{\prime}(t), u^{\prime \prime}(t)\right)\right.
\end{array}\right]=(I-Q) N_{\lambda}(u, v)
$$

Thus,Definition 2.2(d)holds. Therefore, $N_{\lambda}$ is $M$-quasi-compact in $\bar{\Omega}$. The proof is completed.

Theorem 3.1 Assume that the following conditions hold:

$\left(H_{3}\right)$ There exist nonnegative constants $K_{1}, K_{2}$ such that one of (1)and (2) holds:

(1)

$$
B_{1} f\left(t, A_{1}, B_{1}, C_{1}\right)>0, \quad t \in[0,1],\left|B_{1}\right|>K_{1}, A_{1}, C_{1} \in R
$$

and

$$
B_{2} g\left(t, A_{2}, B_{2}, C_{2}\right)>0, \quad t \in[0,1],\left|B_{2}\right|>K_{2}, A_{2}, C_{2} \in R
$$


(2)

$$
B_{1} f\left(t, A_{1}, B_{1}, C_{1}\right)<0, \quad t \in[0,1],\left|B_{1}\right|>K_{1}, A_{1}, C_{1} \in R
$$

and

$$
B_{2} g\left(t, A_{2}, B_{2}, C_{2}\right)<0, \quad t \in[0,1],\left|B_{2}\right|>K_{2}, A_{2}, C_{2} \in R
$$

$\left(H_{4}\right)$ There exist nonnegative functions $a_{i}(t), b_{i}(t), c_{i}(t), e_{i}(t) \in L^{1}[0,1], i=1,2$ such that

$$
|f(t, x, y, z)| \leq a_{1}(t) \varphi_{p}(|x|)+b_{1}(t) \varphi_{p}(|y|)+c_{1}(t) \varphi_{p}(|z|)+e_{1}(t)
$$

and

$$
|g(t, x, y, z)| \leq a_{2}(t) \varphi_{p}(|x|)+b_{2}(t) \varphi_{p}(|y|)+c_{2}(t) \varphi_{p}(|z|)+e_{2}(t)
$$

where $\varphi_{q}\left(\left\|a_{i}(t)\right\|_{1}+\left\|b_{i}(t)\right\|_{1}+\left\|c_{i}(t)\right\|_{1},\right)<2^{2-q}$ if $1<p \leq 2 ; \varphi_{q}\left(2^{p-2}\left\|a_{i}(t)\right\|_{1}+2^{p-2}\left\|b_{i}(t)\right\|_{1}+\left\|c_{i}(t)\right\|_{1},\right)<1$ if $p \geq 2$ The boundary value problem (1.1) has at least one solution.

Lemma3.5 Suppose $\left(H_{3}\right)$ and $\left(H_{4}\right)$ hold.Then

$$
\Omega_{1}=\left\{(u, v) \in \operatorname{dom} M \mid M(u, v)=N_{\lambda}(u, v)\right\}
$$

is bounded in $X$.

Proof. For $(u, v) \in \Omega_{1}$, we have $Q N_{\lambda}(u, v)=0$, i.e.

$$
T_{1}\left(\lambda f\left(t, v(t), v^{\prime}(t), v^{\prime \prime}(t)\right)\right)=0, T_{2}\left(\lambda g\left(t, u(t), u^{\prime}(t), u^{\prime \prime}(t)\right)=0\right.
$$

By $H_{3}$, there exist constants $t_{0}, t_{1} \in[0,1]$ such that $\left|u^{\prime}\left(t_{0}\right)\right| \leq K_{2}$ and $\left|v^{\prime}\left(t_{1}\right)\right| \leq K_{1}$. Since $u(t)=\int_{0}^{t} u^{\prime}(s) d s$,

$u^{\prime}(t)=u^{\prime}\left(t_{0}\right)+\int_{t_{0}}^{t} u^{\prime \prime}(s) d s, v(t)=\int_{0}^{t} v^{\prime}(s) d s, v^{\prime}(t)=v^{\prime}\left(t_{1}\right)+\int_{t_{1}}^{t} v^{\prime \prime}(s) d s$, then that

$$
\begin{gathered}
|u(t)| \leq\left\|u^{\prime}\right\|_{\infty},\left|u^{\prime}(t)\right| \leq K_{2}+\left\|u^{\prime \prime}\right\|_{\infty}, \quad t \in[0,1] . \\
|v(t)| \leq\left\|v^{\prime}\right\|_{\infty},\left|v^{\prime}(t)\right| \leq K_{1}+\left\|v^{\prime \prime}\right\|_{\infty}, \quad t \in[0,1] .
\end{gathered}
$$

It follow from $M(u, v)=N_{\lambda}(u, v),\left(H_{4}\right)$ and (3.2)that

$$
\begin{aligned}
\left|u^{\prime \prime}(t)\right| & =\left|\varphi_{q}\left(\int_{0}^{t} \lambda f\left(s, v(s), v^{\prime}(s), v^{\prime \prime}(s)\right) d s\right)\right| \\
& \leq \varphi_{q}\left(\int_{0}^{1} a_{1}(t) \varphi_{p}(|v|)+b_{1}(t) \varphi_{p}\left(\left|v^{\prime}\right|\right)+c_{1}(t) \varphi_{p}\left(\left|v^{\prime \prime}\right|\right)+e_{1}(t) d t\right) \\
& \leq \varphi_{q}\left[\left(\left\|a_{1}\right\|_{1}+\left\|b_{1}\right\|_{1}\right) \varphi_{p}\left(K_{1}+\left\|v^{\prime \prime}\right\|_{\infty}\right)+\left\|c_{1}\right\|_{1} \varphi_{p}\left(\left\|v^{\prime \prime}\right\|_{\infty}\right)+\left\|e_{1}\right\|_{1}\right]
\end{aligned}
$$

If $1<p \leq 2$, by Lemma 2.1,we get

$$
\begin{aligned}
\left|u^{\prime \prime}(t)\right| & \leq \varphi_{q}\left(D_{1}+C_{1} \varphi_{p}\left\|v^{\prime \prime}\right\|_{\infty}\right) \\
& \leq 2^{q-2}\left[\varphi_{q}\left(D_{1}\right)+\varphi_{q}\left(C_{1}\right)\left\|v^{\prime \prime}\right\|_{\infty}\right] \\
& \leq 2^{q-2}\left[\varphi_{q}\left(D_{1}\right)+\varphi_{q}\left(C_{1}\right)\|v\|\right]
\end{aligned}
$$

thus

$$
\left\|u^{\prime \prime}\right\|_{\infty} \leq 2^{q-2}\left[\varphi_{q}\left(D_{1}\right)+\varphi_{q}\left(C_{1}\right)\|v\|\right]
$$

where $C_{1}=\left\|a_{1}\right\|_{1}+\left\|b_{1}\right\|_{1}+\left\|c_{1}\right\|_{1}, D_{1}=\left(\left\|a_{1}\right\|_{1}+\left\|b_{1}\right\|_{1}\right) \varphi_{p}\left(K_{1}\right)+\left\|e_{1}\right\|_{1}$. On the other hand, since $\left|u^{\prime}(t)\right| \leq K_{2}+\left\|u^{\prime \prime}\right\|_{\infty}, t \in$ $[0,1]$,we get $\left\|u^{\prime}\right\|_{\infty} \leq K_{2}+\left\|u^{\prime \prime}\right\|_{\infty}$ since $|u(t)| \leq\left\|u^{\prime}\right\|_{\infty}, t \in[0,1]$,we get $\|u\|_{\infty} \leq K_{2}+\left\|u^{\prime \prime}\right\|_{\infty}$.Thus

$$
\|u\| \leq K_{2}+2^{q-2}\left[\varphi_{q}\left(D_{1}\right)+\varphi_{q}\left(C_{1}\right)\|v\|\right] .
$$

Similarly,

$$
\|v\| \leq K_{1}+2^{q-2}\left[\varphi_{q}\left(D_{2}\right)+\varphi_{q}\left(C_{2}\right)\|u\|\right]
$$

where $C_{2}=\left\|a_{2}\right\|_{1}+\left\|b_{2}\right\|_{1}+\left\|c_{2}\right\|_{1}, D_{2}=\left(\left\|a_{2}\right\|_{1}+\left\|b_{2}\right\|_{1}\right) \varphi_{p}\left(K_{2}\right)+\left\|e_{2}\right\|_{1}$ Take $M_{1}=\max \left\{2^{q-2} \varphi_{q}\left(C_{1}\right), 2^{q-2} \varphi_{q}\left(C_{2}\right)\right\}$, we get

$$
\begin{gathered}
\|u\| \leq K_{2}+2^{q-2} \varphi_{q}\left(D_{1}\right)+M_{1}\|v\|, \\
\|v\| \leq K_{1}+2^{q-2} \varphi_{q}\left(D_{2}\right)+M_{1}\|u\| .
\end{gathered}
$$


Since $\|(u, v)\|=\|u\|+\|v\|$, we get

$$
\|(u, v)\| \leq \frac{K_{1}+K_{2}+2^{q-2} \varphi_{q}\left(D_{1}\right)+2^{q-2} \varphi_{q}\left(D_{2}\right)}{1-M_{1}}
$$

If $p>2$,by Lemma 2.1,we get

$$
\begin{aligned}
\left|u^{\prime \prime}(t)\right| \leq \varphi_{q}\left(D_{3}+\right. & \left.C_{3} \varphi_{p}\left\|v^{\prime \prime}\right\|_{\infty}\right) \leq \varphi_{q}\left(D_{3}\right)+\varphi_{q}\left(C_{3}\right)\left\|v^{\prime \prime}\right\|_{\infty} \\
& \leq \varphi_{q}\left(D_{3}\right)+\varphi_{q}\left(C_{3}\right)\|v\|,
\end{aligned}
$$

thus

$$
\left\|u^{\prime \prime}\right\|_{\infty} \leq \varphi_{q}\left(D_{3}\right)+\varphi_{q}\left(C_{3}\right)\|v\| .
$$

By (3.2),we get

$$
\|u\| \leq K_{2}+\varphi_{q}\left(D_{3}\right)+\varphi_{q}\left(C_{3}\right)\|v\| .
$$

Similarly

$$
\|v\| \leq K_{1}+\varphi_{q}\left(D_{4}\right)+\varphi_{q}\left(C_{4}\right)\|v\| .
$$

where $C_{3}=2^{p-2}\left(\left\|a_{1}\right\|_{1}+\left\|b_{1}\right\|_{1}\right)+\left\|c_{1}\right\|_{1}, D_{3}=2^{p-2}\left(\left\|a_{1}\right\|_{1}+\left\|b_{1}\right\|_{1}\right) \varphi_{p}\left(K_{1}\right)+\left\|e_{1}\right\|_{1}, C_{4}=2^{p-2}\left(\left\|a_{2}\right\|_{1}+\left\|b_{2}\right\|_{1}\right)+\left\|c_{2}\right\|_{1}, D_{4}=$ $2^{p-2}\left(\left\|a_{2}\right\|_{1}+\left\|b_{2}\right\|_{1}\right) \varphi_{p}\left(K_{2}\right)+\left\|e_{2}\right\|_{1}$, Take $M_{2}=\max \left\{\varphi_{q}\left(C_{3}\right), \varphi_{q}\left(C_{4}\right)\right\}$, we get

$$
\|(u, v)\| \leq \frac{K_{1}+K_{2}+\varphi_{q}\left(D_{3}\right)+\varphi_{q}\left(D_{4}\right)}{1-M_{2}}
$$

So we obtain Lemma 3.5.

Remark1 If we take $\|(u, v)\|=\max \{\|u\|,\|v\|\}$,Lemmma 3.5 still holds. We only need (3.3) into (3.4),we can obtain the Lemma.

Lemma3.6 Assume $\left(H_{3}\right)$ holds, then

$$
\Omega_{2}=\{(u, v) \in \operatorname{Ker} M \mid Q N(u, v)=0\}
$$

is bounded in $X$, whereN $=N_{1}$.

Proof. For $(u, v) \in \Omega_{2}$,we have $(u, v)=\left(b_{1} t, b_{2} t\right)$, then $T_{1} f\left(t, b_{2} t, b_{2}, 0\right)=0, T_{2} f\left(t, b_{1} t, b_{1}, 0\right)=0$. By $\left(H_{3}\right)$,we get $\left|b_{1}\right| \leq$ $K_{2},\left|b_{2}\right| \leq K_{1}$.So, $\Omega_{2}$ is bounded.

Proof of theorem3.1 Let $\Omega=\{(u, v) \in X\|(u, v)\|<r\}$, where $r$ is large enough such that $K_{1}+K_{2}<r<+\infty$ and $\overline{\Omega_{1}} \cup \overline{\Omega_{2}} \subset \Omega$.

By lemma3.5 and Lemma3.6,we can get if $(u, v) \in \operatorname{dom} M \cap \partial \Omega$, then $M(u, v) \neq N_{\lambda}(u, v)$, if $(u, v) \in \operatorname{Ker} M \cap \partial \Omega$, then $Q N(u, v) \neq 0$.

Let

$$
H(u, v, \delta)=\rho \delta(u, v)^{L}+(1-\delta) J Q N(u, v)^{L} . \delta \in[0,1],(u, v) \in \operatorname{Ker} M \cap \bar{\Omega} .
$$

where $J: \operatorname{ImQ} \rightarrow$ KerM is a homeomorphism with $J\left(0,0, b_{1}, b_{2}\right)^{L}=\left(b_{2} t, b_{1} t\right)^{L}$,

$$
\begin{gathered}
\rho=\left\{\begin{aligned}
-1, & \text { if }\left(H_{3}\right)(1) \text { holds } \\
1, & \text { if }\left(H_{3}\right)(2) \text { holds }
\end{aligned}\right. \\
\operatorname{sgn}(x)=\left\{\begin{aligned}
1, & x>0 \\
-1, & x<0
\end{aligned}\right.
\end{gathered}
$$

For $(u, v) \in \operatorname{KerM} \cap \partial \Omega$,we have $(u, v)=\left(b_{1} t, b_{2} t\right) \neq(0,0)$

$$
H(u, v, \delta)=\rho \delta\left(\begin{array}{c}
b_{1} t \\
b_{2} t
\end{array}\right)+(1-\delta)\left(\begin{array}{l}
-T_{2} g\left(t, b_{1} t, b_{1}, 0\right) t \\
-T_{1} f\left(t, b_{2} t, b_{2}, 0\right) t
\end{array}\right)
$$

If $\delta=1, h(u, v, 1)=\rho\left(b_{1} t, b_{2} t\right)^{L} \neq(0,0)^{L}$.If $\delta=0, h(u, v, 0)=J Q N\left(b_{1} t, b_{2} t\right)^{L} \neq(0,0)^{L}$. For $0<\delta<1$, we now prove that $H(u, v, \delta) \neq(0,0)^{L}$.Otherwise, If $H(u, v, \delta)=(0,0)^{L}$, then

$$
\left(\begin{array}{c}
T_{2} g\left(t, b_{1} t, b_{1}, 0\right) \\
T_{1} f\left(t, b_{2} t, b_{2}, 0\right)
\end{array}\right)=\left(\begin{array}{c}
\frac{\rho \delta}{1-\delta} b_{1} \\
\frac{\rho \delta}{1-\delta} b_{2}
\end{array}\right)
$$


Since $\|(u, v)\|=r>K_{1}+K_{2}$, we have $\left|b_{1}\right|>K_{2}$ or $\left|b_{2}\right|>K_{1}$, If $\left|b_{2}\right|>K_{1}$ we have

$$
\begin{gathered}
T_{1}\left(b_{2} f\left(t, b_{2} t, b_{2}, 0\right)\right)=b_{2} T_{1}\left(f\left(t, b_{2} t, b_{2}, 0\right)\right)=\frac{\rho \delta}{1-\delta} b_{2}^{2} \\
\operatorname{sgn}\left(T_{1}\left(b_{2} f\left(t, b_{2} t, b_{2}, 0\right)\right)\right)=\operatorname{sgn}\left[b_{2} f\left(t, b_{2} t, b_{2}, 0\right)\right]=\operatorname{sgn}\left(\frac{\rho \delta}{1-\delta} b_{2}^{2}\right)=\operatorname{sgn}(\rho)
\end{gathered}
$$

if $\left|b_{1}\right|>K_{2}$,we have

$$
\begin{gathered}
T_{2}\left(b_{1} g\left(t, b_{1} t, b_{1}, 0\right)\right)=b_{1} T_{2}\left(g\left(t, b_{1} t, b_{1}, 0\right)\right)=\frac{\rho \delta}{1-\delta} b_{1}^{2} \\
\operatorname{sgn}\left(T_{2}\left(b_{1} g\left(t, b_{1} t, b_{1}, 0\right)\right)\right)=\operatorname{sgn}\left[b_{1} g\left(t, b_{1} t, b_{1}, 0\right)\right]=\operatorname{sgn}\left(\frac{\rho \delta}{1-\delta} b_{1}^{2}\right)=\operatorname{sgn}(\rho)
\end{gathered}
$$

This is a contradiction with the definition of $\rho$. So, $H(u, v, \delta) \neq 0,(u, v) \in \operatorname{KerM} \cap \partial \Omega, \delta \in[0,1]$.

By the homotopy of degree ,we get $\operatorname{deg}(J Q N, \Omega \cap \operatorname{KerM}, 0)=\operatorname{deg}(H(\cdot, \cdot, 0), \Omega \cap \operatorname{Ker} M, 0)=\operatorname{deg}(H(\cdot, \cdot, 1), \Omega \cap \operatorname{Ker} M, 0)=$ $\operatorname{deg}(\rho I, \Omega \cap \operatorname{Ker} M, 0) \neq 0$ By Theorem 2.1,we find that (1.1) has at least one solution in $\bar{\Omega}$.The proof is completed.

Remark2 If $\|(u, v)\|=\max \{\|u\|,\|v\|\}$,Lemma 3.6 still holds. We can take $\max \left\{K_{1}, K_{2}\right\}<r<\infty$, we still get the Theorem 3.1 .

\section{Example}

Let us consider the following boundary value problem

$$
\left\{\begin{array}{l}
\left(\varphi_{p}\left(u^{\prime \prime}\right)\right)^{\prime}(t)=\frac{t^{3}}{4} \sin x^{5}+\frac{1}{8} y^{5}+\frac{t^{2}}{4} \sin z^{5}+\cos t \\
\left(\varphi_{p}\left(v^{\prime \prime}\right)\right)^{\prime}(t)=\frac{t^{4}}{8} \cos x^{5}+\frac{1}{16} y^{5}+\frac{t^{3}}{12} \cos z^{5}+\sin t \\
u(0)=u^{\prime \prime}(0)=0, v(0)=v^{\prime \prime}(0)=0 \\
u^{\prime}(1)=\int_{0}^{1} 2 t u^{\prime}(t) d t, v^{\prime}(1)=\int_{0}^{1} 3 t^{2} v^{\prime}(t) d t
\end{array}\right.
$$

where $p=6$.

Corresponding to problem(1.1),we have $q=\frac{6}{5}, a_{1}(t)=\frac{t^{3}}{4}, b_{1}(t)=\frac{1}{8}, c_{1}(t)=\frac{t^{2}}{4}, e_{1}(t)=\cos t, k_{1}(t)=2 t, a_{2}(t)=\frac{t^{4}}{8}$, $b_{2}(t)=\frac{1}{16}, c_{2}(t)=\frac{t^{3}}{12}, e_{1}(t)=\sin t, k_{2}(t)=3 t^{2}$. Take $K_{1}=2, K_{2}=3$,we can get $\left(H_{1}\right)-\left(H_{4}\right)$ hold.By Theorem3.1,we have the problem(4.1)has at least one solution.

\section{References}

DelPino, M, Elgueta, M, Mansevich, R. (1997). A homotopic deformation along p of a Leray-Schauder degree result and existence for $\left(\left|u^{\prime}\right|^{p-2} u^{\prime}\right)^{\prime}+f(t, u)=0, u(0)=u(T)=0, p>1$. J.Differ.Equ., 80(1), 3227-3238.

Du, Z, Lin, X, \& Ge, W. (2005). Some higher-order multi-point boundary value problem at resonance. J.Comput.Appl.Math., 177, 55-65. http://dx.doi.org/10.1016/j.cam.2004.08.003

Feng, W, \& Webb, JRL. (1997). Solvability of m-point boundary value problems with nonlinear growth. J.Math.Anal.Appl., 212, 467-480. http://dx.doi.org/10.1006/jmaa.1997.5520

Garcia-Huidobro, M, Manasevich, R, \& Zanolin, F. (1994). A Fredholm-like result for strongly nonlinear second order ODEs. J.Differ.Equ., 114, 132-167. http://dx.doi.org/10.1006/jdeq.1994.1144

Garcia-Huidobro, M, \& Ubilla, P. (1997). Multiplicity of solutions for a class of non linear second order equations. Nonlinear Anal, 28(9), 1509-1520. http://dx.doi.org/10.1016/S0362-546X(96)00014-4

Ge, W, \& Ren, J. (2004). An extension of Mawhin's continuation theorem and its application to boundary value problems with a p-Laplacian. Nonlinear Anal,Theory Methods Appl, 58, 477-488. http://dx.doi.org/10.1016/j.na.2004.01.007

Jiang, W. (2011). The existence of solutions to boundary value problems of fractional differential equations at resonance. Nonlinear Anal., Theory Methods Appl. 74, 1987-1994. http://dx.doi.org/10.1016/j.na.2010.11.005

Jiang, W. (2012). Solvability for a coupled system of fractional differential equations at resonance. Nonlinear Anal.: Real World Appl., 13, 2285-2292. http://dx.doi.org/10.1016/j.nonrwa.2012.01.023

Kosmatov, N. (2008). Multi-point boundary value problems on an unbounded domain at resonance. Nonlinear Anal., 68, 2158-2171. http://dx.doi.org/10.1016/j.na.2007.01.038 
Kosmatov, N. (2010). A boundary value problem of fractional order at resonance. Electron. J.Differ.Equ., 135.

Liu, B. (2003). Solvability of multi-point boundary value problem at resonance(II). Appl.Math.Comput., 136, $353-377$. http://dx.doi.org/10.1016/s0096-3003(02)00050-4

Liu, Y, \& Ge, W. (2004). Solvability of nonlocal boundary value problems for ordinary differential equations of higher order. Nonlinear Anal., 57, 435-458. http://dx.doi.org/10.1016/j.na.2004.02.023

Mawhin, J. (1979). Topological degree methods in nonlinear boundary value problems. In: NSFCBMS Regional Conference Series in Mathematics. Am.Math.Soc., Providence. http://dx.doi.org/10.1090/cbms/040

Sun, W, \& Ge, W. (2002). The existence of solutions to Sturm-Liouville BVPs with Laplacian-like operator. ActaMath.Appl.Sin., 18(2), 341-348. http://dx.doi.org/10.1007/s102550200034

Weihua, Jiang. (2014). Solvability of boundary value problem with p-Laplacian at resonance. Boundary value problems, 36.

Zhang, X, Feng, M, \& Ge, W. (2009). Existence result of second-order differential equations with integral boundary conditions at resonance. J.Math.Anal.Appl., 353, 311-319. http://dx.doi.org/10.1016/j.jmaa.2008.11.082

\section{Copyrights}

Copyright for this article is retained by the author(s), with first publication rights granted to the journal.

This is an open-access article distributed under the terms and conditions of the Creative Commons Attribution license (http://creativecommons.org/licenses/by/3.0/). 